\title{
The Fuzzy-Spatial Descriptor for the Online Graphic Recognition: Overlapping Matrix Algorithm
}

\author{
Noorazrin Zakaria ${ }^{1}$, Jean-Marc Ogier ${ }^{1}$, and Josep Llados ${ }^{2}$ \\ ${ }^{1}$ L3i, University of La Rochelle, France \\ \{nzakaria, jmogier\}@univ-lr.fr \\ ${ }^{2}$ CVC, University Autonoma of Barceleno, Spain \\ Josep.Lllados@uab.es
}

\begin{abstract}
In this paper we present the algorithm of our fuzzy-spatial descriptor for symbol recognition in on-line sketches. Fuzzy sets are used to enhance this approach to cope with the inherent distortion of freehand drawings. It allows handling some primitive irregularities due to different ways of drawing in complex composite forms. The descriptor provides complete local information of the primitives that consist of only straight lines. This descriptor used the opening angle between primitives and its overlapping properties in 1D projection to describe its local spatial properties. Segmentation by curve and pen-speed properties of each drawing has been used in obtaining our basic primitives. The experiment results show that a part from its rotational-invariant, this descriptor is also robust to multi-scale of segmentation of its primitives.
\end{abstract}

\section{Introduction}

With the arrival of tactile platform like TabletPC, the online graphic recognition has to face new problems driven by newly arising applications. The most natural and convenient way to provide graphics in a system is to draw sketches on the tactile platform, just like the drawing freehand graphics on a piece of paper. However the sketchy drawings are usually not neat in appearance, neither compact in representation and storage, nor easy for systems to understand and apprehend.

The recognition of online graphic objects is different from the recognition of online handwritings. For online handwritings, a recognizer should be able to tolerate different writing styles. However rotational invariance is not crucial for online handwriting because direction variations of input characters are small.

On the other hand, a graphic objects recognizer requires more flexibility in orientation of each input object with a robust tolerance of different ways of object drawings that cope with the inherent distortion of freehand drawings. It must be able to handle some primitive irregularities due to different way of drawing in complex composite forms. For this situation, we proposed our method, called Overlapping Matrix, which is based on fuzzy set theory with spatial properties between primitives as the basic classes. We used straight lines as the primitives of our input. From these straight line input, we used the opening angle and the projection overlaps between two primitives to describe the spatial properties of each primitive. In order to improve computational efficiency during the matching of primitives, an approach using global spatial descrip- 
tor for every primitive is used. We tested this method using the CVC Barcelona dataset (Graphics Recognition 2003), and it performed at 94\% of recognition accuracy.

To begin with, we described few relative works that have been done in Section 2. The details of the algorithm used in this method are developed in Section 3, followed by Section 4 that described the matching process that consists of regrouping all information of descriptors from individual pairs of primitives and classification process. Section 5 showed some experimental results and evaluation. And we conclude with Section 6 with some comments about this contribution.

\section{Related Works}

A large number of representation and recognition techniques have been proposed for sketch recognition using feature based methods. For example, Rubine [1] defined a gesture characterized by a set of eleven geometric attributes and two dynamic attributes, and constructed a linear discriminate classifier for gesture-based interfaces whose weights are learned from a set of training examples. However, Rubine's method is only applicable to single-stroke sketches and sensitive to drawing direction and orientation.

Fonseca et al[2] proposed a method of symbol recognition using fuzzy logic based on a number of rotational invariant global features, such as the smallest convex hull that can be circumscribed around the shape, the largest triangle that can be inscribed in the hull, and the largest quadrilateral that can be inscribed. Because their classification method relies on aggregate features of pen strokes, it might be difficult to differentiate between similar shapes. A benefit of feature-based approaches is that stroke regrouping is not necessary, while their drawback is that they perform typically in basic shapes recognition, such as rectangle and circles.

In addition, many sketch recognition methods are based on stroke segmentation. Most segmentation techniques in image processing target nicely printed symbols and do not scale well to hand-sketched symbols due to the noise and distortion in sketches. As strokes can be segmented in many different ways, the combinatory approaches based on exhaustive search is clearly infeasible for complex sketchy shapes. Temporal information such as pen speed has been recently explored as a means to uncover users' intentions during sketching. Sezgin[3] and later Calhoun[4], used both curvature and speed information in a stroke to locate breakpoints. Saund[5] uses more perceptual context, including local features such as curvature and intersections, as well as global features such as closed paths. These methods focus on the detection of corner (segment) points, which usually help decompose the original ink into basic geometric components such as lines and arcs. All of them use the empirical thresholds to test the validity of an approximation that ultimately leads to the problem of a threshold being too tight or too loose. Furthermore because of the arbitrariness of the drawing it is hard to manipulate these diverse strokes well. Recently, Heloise et al[6] used Dynamic Programming to approximate recursively a digitized curve with a given number of lines and arc segments based on templates. However, they put emphasis upon stroke segmentation for simple sketchy shapes based on all of the ink points.

In our method, we modified the segmentation of strokes using the approach proposed by Calhoun[4], in order to divide the raw data into straight line segments. In addition to 
the work, we introduced points of inflexions together with maximum and minimum points in our raw data of our input. From these points, we obtained the straight line primitives for our spatial descriptor. 4 fuzzy membership functions have been used to classify the opening of every pair of primitives, and 6 fuzzy membership functions have been used to describe its overlapping properties in 1 axis of reference. The final step in the matching process is performed by comparing the spatial descriptor of every primitive from a sketched input, with every primitive from the selected models.

\section{The Overlapping Matrix Algorithm}

The system is composed of three functional units: extraction of graphic primitives, generation of spatial description, indexation and model based matching.

\subsection{Straight Line Primitive Extraction}

Naturally, users tend to slow the pen when making many kinds of intentional discontinuities in the shape. For example, when drawing a rectangle as a single pen stroke, it is natural to slow down at the corners, which are the segment points. Figure 1 shows the speed profile for a typical square. The corners can be easily identified by the low pen speed.

Once the pen speed has been calculated at each point along the stroke, segment points can be found by thresholding the speed. Any point with a speed below the threshold is a segment point. We specify the threshold as fraction of the average speed along the particular pen stroke. If necessary, the user can adjust the threshold to match his or her particular drawing style.

While many intentional discontinuities occur at low pen speed, others do not. For example, when drawing an "S" shape, there may be no slowdown at the transition from one lobe to the other. Similarly, when drawing a "J" shape, there may be no slowdown at the transition from the line to the arc. We can locate these kinds of segment points by examining the curvature of the pen stroke. Segment points occur at locations where the curvature changes sign.

Firstly a polygonal approximation is used to transform the raw data of online input into straight line primitives. This segmentation is computed based on the speed and curvature properties of the input.

Naturally, users tend to slow the pen when making many kinds of intentional discontinuities in the shape. For example, when drawing a rectangle as a single pen
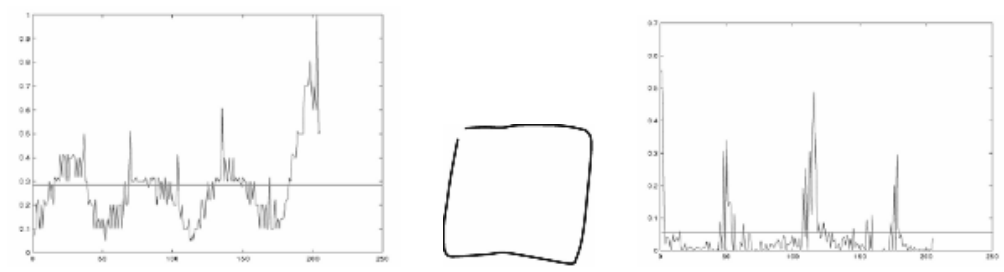

Fig. 1. Speed profile (left) and Curve Profile (right) for a square 
stroke, it is natural to slow down at the corners, which are the segment points. Figure 1 shows the speed profile for a typical square. The corners can be easily identified by the low pen speed.

Once the pen speed has been calculated at each point along the stroke, segment points can be found by thresholding the speed. Any point with a speed below the threshold is a segment point. We specify the threshold as fraction of the average speed along the particular pen stroke. If necessary, the user can adjust the threshold to match his or her particular drawing style.

While many intentional discontinuities occur at low pen speed, others do not. For example, when drawing an "S" shape, there may be no slowdown at the transition from one lobe to the other. Similarly, when drawing a "J" shape, there may be no slowdown at the transition from the line to the arc. We can locate these kinds of segment points by examining the curvature of the pen stroke. Segment points occur at locations where the curvature changes sign.

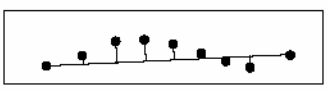

Fig. 2. Window calculating the curvature sign

We consider three distinct signs: positive, negative, and zero. When computing the sign, we examine a window of points on either side of the point in question. We connect the first and last points in the window with a line segment. We then calculate the minimum distance from each point in the window to the line. Distances to the left of the line are positive, while those to the right are negative. Left and right are defined relative to the drawing direction. The signed distances are summed to determine the sign of the curvature.

If the absolute value of the sum is less than a threshold, the curvature is considered to be zero. In the example in Figure 2, the curvature is positive because there are more positive distances than negative ones. (In this example, the drawing direction is from left to right.).

By using a window of points to compute the sign of the curvature, we are able to smooth out noise in the pen signal. Some of the noise comes from minor fluctuations in the drawing, other noise comes from the digitizing error of the input device. The larger the window, the larger the smoothing effect. The size of the window must be tuned to the input device and the user. For mouse input, we have found a window size of $10 \%$ of points with respect to the total points in a stroke to be suitable.

The points of inflexion are also detected by localizing the center of zero curvature between pairs of minimum and maximum points.

Once the strokes have been segmented, the next task is to generate spatial descriptions of each primitive.

This Spatial Descriptor provides structural information of every pairs of primitives in of an online symbol. We used the angle formed between 2 segments in each pair of primitives and its degree of overlapping. In practice, the angle formed between 2 segments and its degree of overlapping are sometimes too ambiguous to be identified. Hence we describe them by assigning a membership degree for every possible case in term of fuzzy set theory. 
On the other hand, The Sequential Descriptor indicates the neighbouring properties of primitives. This is very helpful to account the penalty for the possible errors from bad segmentation of strokes into straight line primitives.

This leads to the descriptor elaborated here-after.

\subsection{Spatial Descriptor}

From straight line primitives, we classified every pairs of primitives according to the angle forming between segments (opening angle) and to the overlapping of one segment to another. Four fuzzy classes have been used to classify the opening angles, whilst 6 classes used to identify the type of overlapping of a segment's 1D projection with respect to another segment.

\subsubsection{Opening Angle}

For every segment $\mathrm{Ni}$, its orientation is defined by,

$$
\theta=\operatorname{arctg}\left[\frac{\left(y_{e}^{\prime}-y_{s}^{\prime}\right)}{\left(x_{e}^{\prime}-x_{s}^{\prime}\right)}\right]
$$

where $\left(x_{s}^{\prime}, y_{s}^{\prime}\right)$ and $\left(x^{\prime}, y_{e}^{\prime}\right)$ are the coordinates of the initial point and the final point of the segment.

For every pair of primitives $\left(N_{a}, N_{b}\right)$, we used the first chosen segment, named $N_{a}$, as a local reference axis. Figure 3 a here-after illustrates this situation.

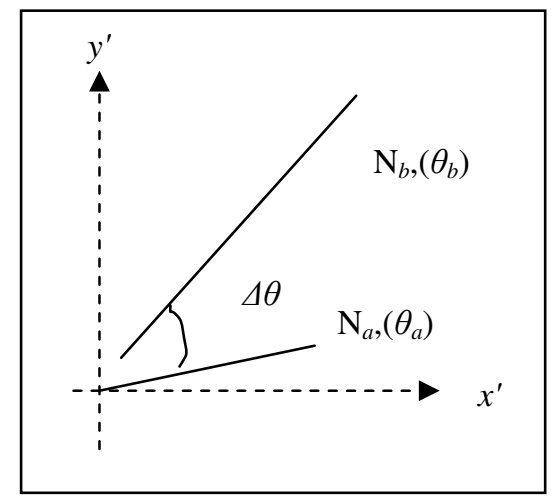

Fig. 3. A pair of primitive

Thus, the angle separating this pair is defined by,

$$
\Delta \theta=\theta_{b}-\theta_{a}
$$

We used 4 classes as fuzzy memberships for this angle. These 4 classes represent 4 possible critical angles for a pair of primitives. They are formulated in a vector,

$$
\left[\mu_{1}(\Delta \theta), \mu_{2}(\Delta \theta), \mu_{3}(\Delta \theta), \mu_{4}(\Delta \theta)\right]
$$

For every opening angle of one pair of primitives, this vector represents the distribution of belonging of the opening angle into those 4 critical angles $\left[0^{\circ}, 45^{\circ}, 90^{\circ}, 135^{\circ}\right]$. 
Table 1. Membership function of the Opening Angles

\begin{tabular}{|c|c|c|c|c|}
\hline Type & $\mu_{1}$ & $\mu_{2}$ & $\mu_{3}$ & $\mu_{4}$ \\
\hline$\Delta \theta\left(^{\circ}\right)$ & $0 \pm \mathrm{k} 180$ & $45 \pm \mathrm{k} 180$ & $90 \pm \mathrm{k} 180$ & $135 \pm \mathrm{k} 180$ \\
\hline
\end{tabular}

In term of fuzzy set theory, the opening angle of a pair of primitives can be regarded as linguistic variable, $\{1,2,3,4\}$ are linguistic terms, each of which corresponds with a fuzzy set, and is the membership function associated with linguistic term $i$, where $i=1,2,3,4$. The membership function of linguistic term $i$ is defined as,

$$
\mu_{i}(\Delta \theta)=\left\{\begin{array}{cc}
1 & \Delta \theta \in\left[\theta_{i}-10^{\circ}, \theta_{i}+10^{\circ}\right] \\
0,5+0,5 \times \frac{\Delta \theta-\left(\theta_{i}-22,5^{\circ}\right)}{12,5^{\circ}} & \Delta \theta \in\left[\theta_{i}-35^{\circ}, \theta_{i}-10^{\circ}\right] \\
0,5+0,5 \times \frac{\left(\theta_{i}+22,5^{\circ}\right)-\Delta \theta}{12,5^{\circ}} & \Delta \theta \in\left[\theta_{i}+10^{\circ}, \theta_{i}+35^{\circ}\right] \\
0 & \Delta \theta \notin\left[\theta_{i}-35^{\circ}, \theta_{i}+35^{\circ}\right]
\end{array}\right.
$$

where $i \in\{1,2,3,4\}$.

\subsubsection{Projection Overlapping}

In every pair of primitives, one segment, $N_{b}$, will be projected into the segment of reference, $N_{a}$. The segment $N_{a}$ will be considered as the new X co-ordinate. The detailed implementation is as follows.

Firstly, the endpoints of all primitives $\left\{N_{b} \mid b=1,2, \ldots, L\right\}$ are transformed from original co-ordinates $\left(x^{\prime}, y^{\prime}\right)$ to a new co-ordinates $(x, y)$ via the well known equation

$$
\begin{aligned}
& x=x^{\prime} \cos (\Delta \theta)+y^{\prime} \sin (\Delta \theta) \\
& y=-x^{\prime} \cos (\Delta \theta)+y^{\prime} \cos (\Delta \theta)
\end{aligned}
$$

where $\Delta \theta$ is the opening angle of pair $\left(N_{a}, N_{b}\right)$ in the old co-ordinates so as to let $N_{a}$ act as the $\mathrm{X}$ axis of the new co-ordinates. Hence, the overlapping is calculated by projecting the segment $N_{a}$ into the new co-ordinate (X axis).

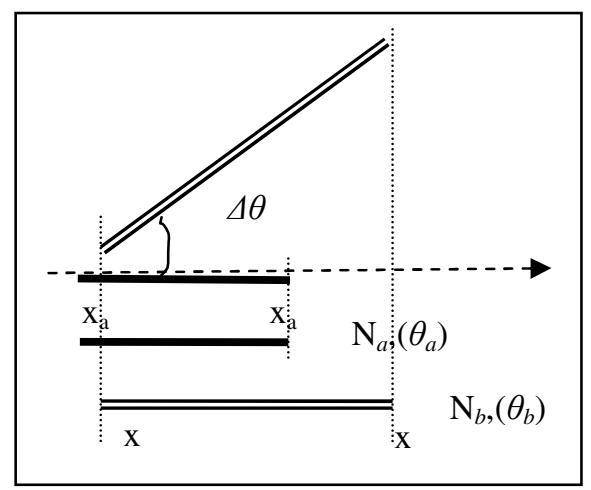

Fig. 4. Projection of segment $N_{b}$ on $N_{a}$ 
Figure 4 above shows the overlapping of the projection of $\mathrm{N}_{\mathrm{b}}\left[\mathrm{x}_{\mathrm{b} 1}, \mathrm{x}_{\mathrm{b} 2}\right]$ with $\mathrm{Na}$ $\left[\mathrm{x}_{\mathrm{a} 1}, \mathrm{x}_{\mathrm{a} 2}\right]$, where $\mathrm{x}_{\mathrm{a} 1}<\mathrm{x}_{\mathrm{a} 2}$ et $\mathrm{x}_{\mathrm{b} 1}<\mathrm{x}_{\mathrm{b} 2}$.

We used 6 fuzzy classes to represent the overlapping of every projection axis of segments. We use a vector

$$
\varepsilon_{i}\left(N_{a}, N_{b}\right)=\left[\begin{array}{l}
\varepsilon_{1}\left(x_{a 1}, x_{a 2}, x_{b 1}, x_{b 2}\right) \\
\varepsilon_{2}\left(x_{a 1}, x_{a 2}, x_{b 1}, x_{b 2}\right) \\
\varepsilon_{3}\left(x_{a 1}, x_{a 2}, x_{b 1}, x_{b 2}\right) \\
\varepsilon_{4}\left(x_{a 1}, x_{a 2}, x_{b 1}, x_{b 2}\right) \\
\varepsilon_{5}\left(x_{a 1}, x_{a 2}, x_{b 1}, x_{b 2}\right) \\
\varepsilon_{6}\left(x_{a 1}, x_{a 2}, x_{b 1}, x_{b 2}\right)
\end{array}\right]
$$

to describe the possibility that an overlapping belongs to every class. In term of fuzzy set theory, type of overlapping can be regarded as linguistic variable, $\{1,2,3,4,5,6\}$ are linguistic terms, and $\varepsilon_{\mathrm{i}}\left(\mathrm{x}_{\mathrm{a} 1}, \mathrm{x}_{\mathrm{a} 2}, \mathrm{x}_{\mathrm{b} 1}, \mathrm{x}_{\mathrm{b} 2}\right)$ is the membership function associated with linguistic term $\mathrm{i}$, where $\mathrm{i}=1,2,3,4,5,6$.

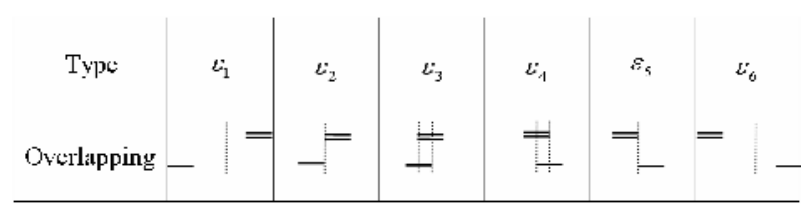

Fig. 5. Membership functions of the overlapping

As illustrated in Figure 5 above, there are 6 types of overlapping between 2 primitives in an $\mathrm{X}$-axis projection. We used a linear distribution for those 4 fuzzy linguistic terms. Figure 5 also shows us that the overlapping properties of $\varepsilon_{1}, \varepsilon_{2}$ and $\varepsilon_{3}$ reflect those of $\varepsilon_{4}, \varepsilon_{5}$ and $\varepsilon_{6}$.

To begin with, we describe here-below the overlapping types $\varepsilon_{2}$ and $\varepsilon_{5}$. These two classes represent a total separation of each primitive in a pair of segments that projected into a new axis of reference. The limit inferior of these classes is when the primitives in this pair touch each other. During the overlapping, these classes represent the relative length of a fraction of a projection (of the segment $N_{b}$ for $\varepsilon_{2}$, and $N_{a}$ for $\varepsilon_{5}$.) that is not overlapped.

For the fuzzy membership function of $\varepsilon_{2}$, the projection of $N_{a}$ is situated on the left of $N_{b}$. This function is defined as:

$$
\varepsilon_{2}=\left\{\begin{array}{cc}
1-\varepsilon_{1} & x_{a 2} \leq x_{b 1} \\
\frac{\left(x_{b 1}-x_{a 1}\right)}{a_{\max }} & \left(x_{b 1}<x_{a 2}\right) \wedge\left(x_{b 1}>x_{a 1}\right) \\
0 & \text { else }
\end{array}\right.
$$


On the other hand, for the fuzzy membership function of $\varepsilon_{5}$, the segment $N_{b}$ situated on the left of the projection of $N_{a}$. Thus, its membership function is defined as:

$$
\varepsilon_{5}=\left\{\begin{array}{cc}
1-\varepsilon_{6} & \left(x_{a 1} \geq x_{b 2}\right) \\
\frac{\left(x_{a 2}-x_{b 2}\right)}{a_{\max }} & \left(x_{a 1}<x_{b 2}\right) \wedge\left(x_{a 2}>x_{b 2}\right) \\
0 & \text { else }
\end{array}\right.
$$

where $\mathrm{a}_{\max }=\left(\mathrm{x}_{\mathrm{a} 2}-\mathrm{x}_{\mathrm{a} 1}\right)$ and $\mathrm{b}_{\max }=\left(\mathrm{x}_{\mathrm{b} 2}-\mathrm{x}_{\mathrm{b} 1}\right)$, represent the total length of segment $N_{a}$ and the projection length of $N_{b}$ on new x-axis that is parallel with $N_{a}$. The first condition in equations (7) and (8) above, represents a total separation of those projections. The second condition shows the relative length of segment of reference $\left(N_{a}\right)$ that is not overlapped. The function returns zero elsewhere.

For the fuzzy membership function of $\varepsilon_{3}$ and $\varepsilon_{4}$, a linear distribution is assured by integrating a relative distance of projections' submerge, named $\varepsilon$ ', together with a distance between the centers of two overlapping projections.

We divided the compute of the relative distance of $\varepsilon$ ' into 4 situations of overlapping:

1. The first situation consists of a partial overlapping, where one of the extreme points of every projection stay outside the overlapping. During this type of overlapping, the membership functions of $\varepsilon$ ', represent a ratio between the length of the overlapped projections and the length of segment used as axis of reference, $N_{a}$.

2. The second situation is described by a projection that submerges totally in the projection of the segment that used as X-axis. This happens when the projection of $N_{b}$ is smaller than $N_{a}$.

3. The third situation is the opposite of the one that describe in the second. The projection of $N_{b}$ is longer than $N_{a}$.

4. And the forth situation is the complement of the first one. It represents the same ratio as explained in the first situation above.

For the function of $\varepsilon_{3}$ and $\varepsilon_{4}$, these 4 situations are represented by:

$$
\mathcal{E}^{\prime}=\left\{\begin{array}{cc}
\frac{\left(x_{a 2}-x_{b 1}\right)}{a_{\max }} & \left(x_{a 2}>x_{b 1}\right) \wedge\left(x_{a 2}<x_{b 2}\right) \wedge\left(x_{a 1}<x_{b 1}\right) \\
\frac{1}{2} & \left(x_{b 2}>x_{a 2}\right) \wedge\left(x_{a 1}<x_{b 1}\right) \\
\frac{1}{4} & \left(x_{b 1}<x_{a 1}\right) \\
\frac{\left(x_{b 2}-x_{a 1}\right)}{a_{\max }} & \text { else }
\end{array}\right.
$$

After considering all possibilities of overlapping, we defined the 2 central membership function, $\varepsilon_{3}$ and $\varepsilon_{4}$, by multiplying the submergence relative, $\varepsilon$ ', with the relative distance of centers between 2 overlapping projections. 
The relative distance of those centers is defined by:

$$
r_{\text {centre }}=\frac{2 \cdot\left|m_{b}-m_{a}\right|}{a_{\max }+b_{\max }}
$$

Hence, we defined our middle membership functions by:

$$
\begin{gathered}
\varepsilon_{3}=\varepsilon^{\prime} \cdot\left(r_{\text {centre }}+1\right) \\
\varepsilon_{4}=\varepsilon^{\prime} \cdot\left(1-r_{\text {centre }}\right)
\end{gathered}
$$

Last but not least, we introduced our last 2 marginal membership functions in order to differentiate between a limit of separation $\left(\varepsilon_{2}\right.$ and $\left.\varepsilon_{5}\right)$ and a total separation within a restricted window of descriptor $\left(\varepsilon_{1}\right.$ and $\left.\varepsilon_{6}\right)$.

Thus, our marginal membership functions are defined by:

$$
\begin{gathered}
\varepsilon_{1}=\left\{\begin{array}{cc}
\frac{\left(x_{b 1}-x_{a 2}\right)}{\text { Dist }_{P M S}} & x_{b 1}>x_{a 2} \\
0 & \text { else }
\end{array}\right. \\
\mathcal{E}_{6}=\left\{\begin{array}{cc}
\frac{\left(x_{a 1}-x_{b 2}\right)}{\text { Dist }} & x_{a 1}>x_{b 2} \\
0 & \text { else }
\end{array}\right.
\end{gathered}
$$

where Dist ${ }_{\text {PMS }}=$ window width $-\left(\mathrm{b}_{\max }\right)-\left(\mathrm{a}_{\max }\right)$

At the end of this process, we have the overlapping between 2 primitives $N_{a}$ and $N_{b}$ in 1 dimentional plan can be obtained by synthesizing their overlapping on $\mathrm{X}$-axis (segment $N_{a}$ ). Here, we let:

$$
\varepsilon_{(i)}\left(N_{a}, N_{b}\right)=\varepsilon_{i}\left(x_{a 1}, x_{a 2}, x_{b 1}, x_{b 2}\right)
$$

\subsection{Reconstruction of Spatial Descriptor}

Our spatial descriptor for every pair of primitives is defined by:

$$
D s_{k,(i)}\left(N_{a}, N_{b}\right)=\mu_{k}(\Delta \theta) \times \varepsilon_{(i)}\left(N_{a}, N_{b}\right)
$$

For every pair of primitives, we obtained a matrix of $(4 \times 6)$ elements that describes the spatial properties between them. We applied this descriptor to all possible combination of every pairs of primitives.

\section{Matching Process}

For every input of online symbols, we have a list of descriptor matrices that describe all possible combination of primitive relationships. In a matching process, we can obtain a very precise description of primitives by comparing all descriptor matrices individually. However, this locally rich information from our descriptor requires lots of time to compute those matrices individually. 


\subsection{Global Approach}

We improved this matching process by regrouping all local information of all pairs according to the segments used as reference axis. To do so, we added all descriptor matrices that belong to the same segment of reference. As a result, we obtained another global spatial descriptor of every primitive. The global spatial descriptor of each primitive $N_{p}$ is defined by:

$$
D S\left(N_{p}\right)=\frac{\sum_{q=1}^{L} D s_{k,(i)}\left(N_{p}, N_{q}\right) \cdot w_{p q}}{\sum_{q=1}^{L} w_{p q}}
$$

where $L$ represent the total number of primitives, $w_{p q}$ represent the weigh that used to describe the sequence of 2 primitives.

For 2 consecutive primitives, we reduce the weight of $w_{p q}$ so that the global primitive descriptor is less influenced from them. At the same time, $w_{p q}$, is also proportional to the relative distance between the reference segment and the projection of its pair. Hence we improved its robustness to noises due to bad segmentation.

\subsection{Matching}

With the global approach, our primitive descriptor provides information of spatial and relational relationship of each primitive of an online graphic form. The matching between the input and models is to find the correspondence between primitives. This can be done by computing the nearest similarity.

Suppose that an input symbol is composed by line segments $w=\left\{N_{p} \mid p=1,2, \ldots, L\right\}$ and the model to be compared consists of line segments $w^{\prime}=\left\{N^{\prime}{ }_{q} \mid q=1,2, \ldots, L^{\prime}\right\}$. The similarity distance between these two line segments $N_{p}$ and $N^{\prime}{ }_{q}$ is defined as:

$$
d\left(N_{p}, N_{q}^{\prime}\right)=\sum_{k=1}^{4} \sum_{i=1}^{6}\left|D s_{k, i i}\left(N_{p}\right)-D s_{k,(i)}\left(N_{q}^{\prime}\right)\right|
$$

where $D s_{k,(i)}\left(N_{p}\right)$ and $D s_{k,(i)}\left(N_{q}^{\prime}\right)$, defined in equation (18), are the statistical feature associated with each line segment.

Matching two symbols is to find the correspondence between their line segments. This can be done using the nearest neighbour. Following is the computation:

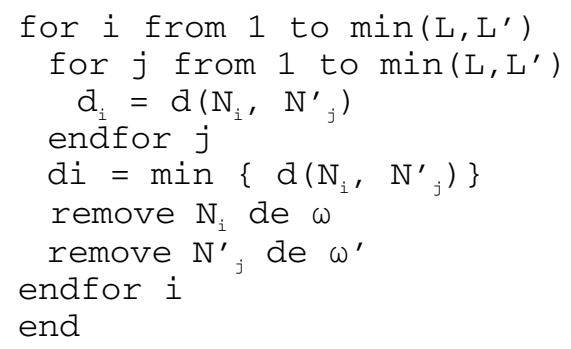

For every loop of the computation above, we omitted segment from the input, $N_{p}$, that similar to the one from the model, $N_{p}{ }_{p}$. At the end of these iterations, we obtained 
$\min \{\mathrm{L}, \mathrm{L}$ ' $\}$ matched pairs and IL-L'l of isolated segments. Thus, the distance between symbols is defined as:

$$
D=\frac{\left(1+\frac{\left|L-L^{\prime}\right|}{L+L^{\prime}}\right) \cdot \sum_{l=1}^{\min \left\{L, L^{\prime}\right\}}\left(d_{l} \cdot w_{l}\right)}{\sum_{l=1}^{\min \left\{L, L^{\prime}\right\}} w_{l}}
$$

where $\sum \mathrm{d}_{1}$ is the cost of $\min \left\{{\left.\mathrm{L}, \mathrm{L}^{\prime}\right\}}\right\}$ matched pairs, $\left[1+\left|\mathrm{L}^{-\mathrm{L}^{\prime}}\right| /\left(\mathrm{L}+\mathrm{L}^{\prime}\right)\right]$ accounts the penalty for $\mid \mathrm{L}-\mathrm{L}$ '। unmatched primitives and $w_{l}$ weighs the similarity distance with respect to the length of the segment.

The objective of fuzzy matrix algorithm is to find the nearest neighbour for an input sketch within selected models. Based on equation (18), the distance between every candidate model and the input symbol can be determined. Then, the model with the minimum distance to the input sketch is selected as the retrieved model.

\section{Experimental Results}

A dataset collected manually from CVC Barcelona, is used for the comparison. It consists of 50 classes of architectural online and offline symbols, drawn by 40 different people and 20 instances each. Our retrieval is focused in comparing an on-line symbol towards its perfect off-line model of reference.

The matching accuracy of our approach improved up to $94 \%$ compared to $88 \%$ initially when we did not introduce the sequential factor and segment length in our descriptor. The experimental results also show that this method sustains the same performance of model retrieval with different ways of sketching the strokes.

However, the drawback of our approach is due to its run time of recognition, where it has a tendency of increasing proportionate to the number of primitives. It remains acceptable to isolated symbols that have limited number of segments.

\section{Conclusion}

In this paper, we propose a complete fuzzy-spatial descriptor in online graphic recognition, proven to be robust in rotational and primitive sequences. The integration of feedbacks in final classification coupled with intelligent matching of structural descriptors is our future endeavour.

\section{References}

1. Rubine Dean, Specifying gestures by examples, Computer Graphics, 1991, 25:329-337.

2. Fonseca M. J., Pimentel C. and Jorge J. A., CALI - an online scribble recognizer for calligraphic interfaces. In AAAI Spring Symposium on Sketch Understanding, AAAI Press (2002) 51-58.

3. Sezgin T. M., Stahovich T., Davis R., Sketch based interfaces: early processing for sketch understanding. In Proceedings of the 2001 Workshop on PUI, Orlando, Florida, (2001) 1-8. 
4. Calhoun C., Stahovich T. F., Kurtoglu T. and Kara L. B., Recognizing multi-stroke symbols. In Proceddings of 2002 AAAI Spring Symposium - Sketch Understanding, AAAI Press (2002) 15-23.

5. Saund E., Finding Perceptually Closed Paths in Sketches and Drawings. IEEE Transactions on Pattern Analysis and Machine Intelligence. 25(4), (2003) 457-491.

6. Heloise H., Micheal S., and Richard N., Robust Sketched Symbol Fragmentation using Templates. International Conference on Intelligent User Interface, ACM Press 156-160. 